\title{
Wissenstransfer in medizinischen Internetforen Am Beispiel des Akustikusneurinoms
}

\author{
Elisabetta LONGHI
}

\begin{abstract}
The transfer of knowledge in medical internet forums, using the example of acoustic neuroma

More and more patients are seeking advice on medical internet forums, which give them the chance to communicate easily with other laypeople as well as with doctors. Examining a forum on acoustic neuroma, this paper investigates the features of this specific type of medical discourse, in particular how the medium influences the way of communicating information, as well as the changing role of laypeople and moderators in the online environment.
\end{abstract}

Keywords: transfer of knowledge, internet forums, medical discourse, informed laypeople, acoustic neuroma

DOI: doi.org/10.15452/StudiaGermanistica.2021.28.0003

\section{Einleitung}

Internetforen sind eine internetbasierte Kommunikationsform, die sich seit ihrem Entstehen großer Beliebtheit erfreut: Bereits 2008 zählte Michael Ebner (vgl. Ebner 2008:9) allein im deutschsprachigen Raum mehrere Tausend und heute kann von einem weiteren Zuwachs ausgegangen werden. Dieser große Erfolg ist nicht zuletzt auf die bidirektionale bzw. multidirektionale Interaktion unter den Forumsteilnehmern zurückzuführen, die zugleich Textrezipienten und -Produzenten sein können, wie in einem räumlich und zeitlich versetzten Gespräch (mehr dazu unter 6.3).

Die Etymologie spricht für sich: Das Wort „Forum“ stammt aus dem Lateinischen und bedeutet ursprünglich Marktplatz auch im Sinne von Versammlungsort, nicht unbedingt nur zu kommerziellen Zwecken. Analog zu den römischen Foren bilden auch die modernen, webbasierten Internetforen einen Treffpunkt für Unterhaltungen über die verschiedensten Themen (vgl. Czauderna 2014:20; Born 2010:172).

Unter Internetforen versteht Ebner (2008) ebenso wie Hass (vgl. 2014:17) einfach Diskussionsforen, als ob es sich hierbei um Synonyme handeln würde. Eigentlich sind diese Bezeichnungen jedoch nicht völlig gleichzusetzen, vielmehr bildet „Internetforen“ eher einen Oberbegriff, in dem das Bestimmungswort lediglich das Kommunikationsmedium nennt und nicht auf die differenzierten Anforderungen an ein solches Medium eingeht. Innerhalb der Internetforen lässt sich näher betrachtet zwischen Diskussions- und Hilfeforen unterscheiden (vgl. Ehrhardt 2009:118 f.), wenn man 
die teils divergierenden Zwecke berücksichtigt. Vereinfacht dargestellt dienen Diskussionsforen dem Meinungsaustausch unter Menschen mit ähnlichen Interessen, Hilfeforen dagegen primär dem Informationsaustausch zugunsten von Ratsuchenden, die ein Problem haben.

Medizinische Internetforen sind in der Regel Hilfeforen, die es Patienten ermöglichen, sich mit anderen Laien und/oder mit Fachexperten in Verbindung zu setzen, wobei die fragestellenden Mitglieder von ihren Kommunikationspartnern kompetente Ratschläge oder sogar eine fachliche Beratung erwarten. Das Internetforum wird somit zu einer nicht institutionalisierten Online-Arztpraxis.

\section{Medizin im Netz}

Abgesehen von den zahlreichen Ressourcen für Profis, von Fachzeitschriften bis hin zu medizinischen Datenbanken, bietet das Netz eine Menge an populärwissenschaftlichen Inhalten im Gesundheitsbereich. Der Laie, der Informationen einholen möchte, steht vor einer großen Auswahl und nutzt diese auch gerne.

Im Mai 2010 wurde vom Marktforschungsinstitut Skopos eine Umfrage durchgeführt, aus der sich ergab, dass sich fast 80 Prozent der Befragten über Gesundheitsfragen im Netz informierten (vgl. ,Deutsches Ärzteblatt‘, 30/2010). Heute, mehr als 10 Jahre später, wäre ein noch höherer Prozentsatz nicht wunderlich. Mittlerweile sind auch virtuelle Sprechstunden per E-Mail, Telefon oder Videochat gängig geworden (vgl. ,Deutsches Ärzteblatt‘, 47/2012 sowie ,ÄrzteZeitung' 2020) und neben kostenpflichtigen Dienstleistungen bieten Ärzte nicht selten unentgeltliche Beratungen an: Wieso sie es tun bzw. tun sollen, erklärt wieder das ,Deutsche Ärzteblatt' mit einem expliziten Verweis auf Internetforen. Da diese laut der genannten Umfrage bereits 2010 angeblich von circa 40 Prozent der Patienten besucht wurden (vgl. ,Deutsches Ärzteblatt‘, 45/2010), bestehe die reelle Gefahr, dass Fake-News im medizinischen Bereich verbreitet werden, was negative Auswirkungen auf die alltägliche ärztliche Praxis haben könne, so das ,Deutsche Ärzteblatt', das ganz ausdrücklich vor „selbsternannten Experten“ warnt. „Deshalb ist es ein Gebot der Vernunft, wenn Ärzte sich als kompetente Teilnehmer an seriösen Foren betätigen, dort fachkundig antworten und Fehlinformationen zurechtrücken“ (,Deutsches Ärzteblatt”, 45/2010).

Die gravierende Frage, was seriöse Foren ausmacht und sie von unseriösen abgrenzt, ist nicht leicht zu beantworten, nicht zuletzt, weil oft keine scharfe Trennlinie gezogen werden kann. Sicher herrscht eine große Vielfalt an medizinischen Internetforen und das auffälligste Klassifikationskriterium ist das thematische Spektrum.

\section{Was für ein Wissen?}

\subsection{Themenkreis}

Die bekanntesten deutschsprachigen Foren, die im Folgenden aufgelistet sind, umfassen das gesamte Fachgebiet der Medizin:

- das Deutsche Medizin-Forum (URL 1);

- das Forum med1 (URL 2);

- das Forum von DocInsider (URL 3);

- das Forum der Webseite von Onmeda (URL 4);

- das Unterforum Medizin der Experten- und Ratgeber-Community wer-weiss-was (URL 5).

Andere sind auf bestimmte Themen bzw. Erkrankungen spezialisiert und haben dementsprechend weniger Mitglieder: Normalerweise interessieren sich z. B. für seltene Krankheiten nur hochspezialisierte Fachärzte und diejenigen, die daran leiden oder deren Freunde bzw. Angehörige betroffen sind.

So ist es auch für das IGAN-Forum (URL 6), das dem Akustikusneurinom gewidmet ist, einem seltenen Hirntumor, der zwar langsam wächst und keine Metastasen bildet, aber trotzdem über kurz 
oder lang erst zu lästigen und später zu lebensbedrohenden Beschwerden führen kann. Das IGAN-Forum wird der spezielle Untersuchungsgegenstand des vorliegenden Beitrags sein, weil es einerseits aufgrund seiner textlinguistischen Merkmale exemplarisch wirkt, andererseits fast ein Unikum ist, mit dessen Untersuchung sich zukunftsweisende Tendenzen beobachten lassen.

\subsection{Wissensvertiefung durch das IGAN-Forum Akustikusneurinom}

Das IGAN-Forum fällt in die Kategorie der Online-Selbsthilfegruppen (Kleinke 2015:415-417), „IGAN“ steht nämlich für „Interessengemeinschaft Akustikusneurinom“, eine „freie, nicht organisierte Selbsthilfegruppe [...] ohne Rechtsform, ohne Satzung, ohne formeller [sic!] Beitrittserklärung und ohne Mitgliedsgebühr" [fett im Original], wie in der programmatischen Selbstdarstellung zu lesen ist (URL 7). Der bewusste Verzicht auf jegliche Formalitäten sowie das Fehlen von Werbeanzeigen, die dagegen in vielen anderen Foren grassieren (vgl. ,Deutsches Ärzteblatt', 45/2010), sollen den ungezwungenen Austausch von unabhängigen Informationen fördern.

Das IGAN-Forum ist jedenfalls nie die erste Informationsquelle für die Interessenten, die, wie oben erklärt, entweder Fachleute oder Betroffene sind. Von Seiten ihrer Adressaten können Beitragende immer ein gewisses Vorwissen präsupponieren, denn auch ratsuchende Patienten sind mehr oder weniger informierte Laien, die sich erst nach der Diagnose eines Akustikusneurinoms im Forum informieren. Diese Rahmenbedingungen versprechen schon einen höheren Fachlichkeitsgrad als es normalerweise der Fall ist, was vor allem auf der lexikalischen Ebene deutlich wahrzunehmen ist. Selbst Laien bedienen sich in ihren Beiträgen eines ausgesprochen professionellen Wortschatzes, der durch genaue Fachbezeichnungen für Körperteile, Beschwerden und Therapien sowie im Fach geläufige Abkürzungen gekennzeichnet ist, wie folgende Beispiele veranschaulichen sollen:

- Körperteile > Hirnstamm, Mundast, Schädelbasis, Kleinhirnbrückenwinkel u. v. a.;

- Krankheiten und Beschwerden > intracochleäres Schwannom, Okosklerose (Verknöcherung der Gehörknochen), Facialisparese, Synkinesien, Hypoglossus-Facialis-Anastomose, Tinnitus, Trigeminusneuralgie, Tieftonsenke, Hyporeflexie u. v. a.;

- Therapien > Cross-Face Nerve Graft, also die „Kopplung des n. facialis der gesunden Seite mit dem n. suralis, der vom Unterschenkel entnommen wird“, CyberKnife, Gamma Knife, Reizstromtherapie u. v. a.;

- Abkürzungen > AKN bzw. AN (Akustikusneurinom), OP (Operation), UK (Universitätsklinik), MRT (Magnetresonanztomografie), HNO (Hals-Nasen-Ohren) u. v. a.

Aus dieser Liste wird deutlich, dass sich sogenannte Laien auch nicht vor englischen und lateinischen Fachausdrücken scheuen. Die Erklärungen für Okosklerose und Cross-Face Nerve Graft wurden jeweils von den sehr gut informierten Patientinnen Loddi (14.02.2020) und Lotta88 (06.05.2012) gegeben und solche Strategien der sprachlichen Wissensvermittlung, d.h. anaphorische Kurzdefinitionen anhand von Parenthesen, elliptischen Zwischensätzen und synonymen Dubletten (vgl. Magris 2009:91-96), befinden sich auch in anderen, gleichfalls von Laien verfassten Forumsbeiträgen: Dadurch kommt zum Vorschein, dass sich die Schreibenden der Fachlichkeit bzw. Schwierigkeit mancher Ausdrücke wohl bewusst sind.

Es liegt auf der Hand, dass sie die Fachtermini von fachkundigen Personen übernommen haben, die sie konsultierten, bevor sie sich ans Schreiben machten. Nicht umsonst verweisen die Anführungszeichen bei Lotta88 auf den intertextuellen Charakter der von ihr bloß zitierten Erläuterung zum Cross-Face Nerve Graft. Wie schon angedeutet, ist die Nutzung des IGAN-Forums kein Ersatz für den tatsächlichen Arztbesuch, der in der Regel schon stattgefunden hat. Anders verhält es sich mit anderen medizinischen Internetforen, wo Diagnosen sehr gefragt sind und in ziemlich hohem Maße improvisiert erstellt werden. Gerade die Themen, die bei einem Arztbesuch marginal bleiben, sind im IGAN-Forum besonders häufig vertreten, darunter an erster Stelle die physischen und psychischen Empfindungen der Patienten, die gerne ihre Erfahrungen austauschen. Das sind Informationen, die Ärzte nicht geben können, es sei denn, sie leiden selbst an der Krankheit, weil 
letztendlich nur Betroffene wissen, wie es sich anfühlt, mit einem Akustikusneurinom zu leben: Das kapiert aber kaum ein Nicht-Betroffener (Sina1, 18.02.2019).

Die Situation lässt sich folgendermaßen zusammenfassen: Das von Profis in Präsenz erteilte wissenschaftliche Wissen wird online um von Leidensgenossen geliefertes Alltagswissen ergänzt, von besser informierten Laien laienhaft aufbereitet und mit praktischen Infos (z. B. Namen und Adressen von Ärzten und Kliniken) versehen. Im virtuellen Raum werden auch diejenigen Wissenslücken gefüllt, die in der Arztpraxis aus Zeitmangel entstanden sind: „Ärzte haben heute weniger Zeit für ihre Patienten als vor wenigen Jahren. Die Patienten bleiben oft mit Fragen und Zweifel zurück." (Marc Shaffu:1).

Zeitmangel und ungünstige Bedingungen sind nicht die einzigen Gründe, weshalb sich viele Patienten nach dem Arztbesuch über ihre Krankheit noch nicht im Klaren sind: Manche Fragen werden notwendigerweise erst im Nachhinein gestellt, wenn der erste Schock der unerwarteten Diagnose überwunden ist. Hierzu äußert der Moderator ANFux seine Empfehlungen:

Ich habe vollstes Verständnis für die Gefühle nach Erhalt der Diagnose „,Tumor “ und antworte in diesen Stunden bzw. Tagen auch individuell per VN. Aber bitte - in diesen ersten Stunden ist Besinnen angesagt, Ruhe finden, dann sich informieren und erst dann andere fragen. Und davor die Befragten informieren! (ANFux, 11.02.2012)

Die gut überlegten, passenden Fragen betreffen dann häufig die Therapieentscheidung und die Arztund Klinikauswahl, die schon auf der programmatischen Seite des Forums zu den Hauptthemen zählen. Da die Ärzte in manchen Fällen darüber uneinig sind, ob die Operation, die Bestrahlung oder das sogenannte Warten unter Beobachtung (watch and see) die beste Lösung sei, fühlen sich die Patienten zwangsläufig verunsichert und wollen im Forum die jeweiligen Pros und Kontras abwägen, um eine bewusstere Entscheidung treffen zu können.

\section{Motivation(en)}

Die Einholung einer Zweitmeinung gilt als einer der Hauptgründe für die Beteiligung an einem medizinischen Internetforum sowie im Allgemeinen für die Gesundheitskommunikation im Internet (vgl. u.a. Rossmann/Stehr 2010:347 f.). Das IGAN-Forum ist in dieser Hinsicht keine Ausnahme, denn einige Nutzer äußern ausdrücklich ein solches Bedürfnis, z. B. Loddi und Lotta88:

Nun suche ich nach Erfahrungen und ggf. auch nach weiteren Experten für die Einholung einer Zweitmeinung. (Loddi, 16.02.2020)

Ich nehme an, dass Herr Dr. Eisenhardt sich gut damit auskennt, trotzdem hätte ich gern eine zweite Meinung. Wenn also jemand Erfahrungen diesbezüglich hat oder mir jemanden in diesem Fachgebiet empfehlen kann, bin ich dankbar für jeden Ratschlag. (Lotta88, 06.05.2012)

Die Anonymität der virtuellen Umgebung - die Beiträge werden unter Pseudonymen verfasst - erleichtert auch das Zulassen von starken Emotionen und unbändigen Ängsten, die in der reellen Welt oft verdrängt werden (sollen):

Es ist einfach weit verbreitet Dinge [sic!] herunterzuspielen. Ich erfahre auch aus dem Freundes- und Familienkreis immer wieder Gegenwind und Hypochondervorwürfe. Die Ärzteschaft reiht sich da nahtlos ein. (hatANgst, 04.07.2018)

Vielen Dank für das gute Zureden; mien [sic!] Umfeld kann sich nicht da reinversetzen; entweder sind sie überfordert und können damit nicht umgehen, oder haben totales Mitleid. Wirklich gut, dass ich mich hier nicht alleine fühlen muss. (Plumps, 12.11.2010)

Die bisherigen Untersuchungen zu diesem Thema bestätigen das Bedürfnis nach emotionaler Unterstützung: „Vor allem bei chronischen Krankheiten bietet der medienvermittelte Austausch mit 
ebenfalls Betroffenen mitunter mehr Unterstützungspotenzial als das nahe soziale Umfeld“" (Rossmann/Stehr 2010:350). Das Forum wird moderiert und somit sorgt man auch dafür, dass dieser unrationelle Beweggrund für die Teilnahme am Forum nicht die Oberhand über die rationelle Informationssuche bekommt.

Die Moderatoren sind informierte Laien, die ihre Tätigkeit als eine altruistische Dienstleistung im Namen der Solidaritätsethik verstehen und unter dem IGAN-Motto „Hilfe zur Selbsthilfe“ agieren. Sie sind selbst Betroffene, also Patienten, so wie auch Guido Fluri, der schweizerische Unternehmer, der 2007 die IGAN gründete und ihre Finanzierungen ebenfalls als ,reines humanitäres Engagement" definiert. Auf der Webseite der IGAN stellt sich der Gründer persönlich vor (URL 8) und betont dabei den Wissenstransfer als Hauptzweck der Organisation und ihrer Online-Plattform, in die eben auch das Forum integriert ist.

Da es sich um eine Selbsthilfegruppe handelt, steht die Patientenperspektive im Mittelpunkt, trotzdem sind auch Fachleute in die Initiative involviert. Bemerkenswert ist im Forum die grundsätzlich positive Einstellung - mit nur wenigen Ausnahmen (s. das obige Beispiel) - den Ärzten gegenüber, auch dort, wo nach einer zweiter Meinung gefragt wird, z. B. möchte Lotta88 die fachliche Kompetenz von Dr. Eisenhardt keineswegs anzweifeln, ganz im Gegenteil. Dieses kooperative Patienten-Arzt-Verhältnis ist keine Selbstverständlichkeit, denn in anderen medizinischen Internetforen wird vor allem Kritik gegen vermeintlich inkompetente Ärzte laut (vgl. ,Die Zeit‘, 14/2008).

Die Zusammenarbeit zwischen Experten und Laien setzt die Reziprozität voraus. Der IGAN stehen zwar „führende Fachärzte der Schädelbasischirurgie beratend und unterstützend zur Seite“ (URL 7), so dass die Zuverlässigkeit der vermittelten Informationen gesichert ist, aber ihrerseits finanziert die Organisation Forschungsprojekte zur Diagnose und Therapie des Akustikusneurinoms. Und das ist nicht alles: Sogar die Richtung des Wissenstransfers ist bidirektional.

\section{Wissenstransfer: von wem zu wem?}

Als Hilfeforum ist das IGAN-Forum eigenartig, wenn man bedenkt, dass der Status der Beitragenden hier sehr fließend ist und die herkömmliche Dichotomie von Ratsuchenden und Ratgebern dadurch aufgehoben wird. Fandrych/Thurmair (2011:250) beschreiben die für Beratungstexte typische Situation auf folgende Weise:
„Allen beratenden Texten [...] gemeinsam ist die Grundkonstellation, dass sich aufgrund einer ko- operativen Basis ein Ratsuchender an eine zweite Person mit der Bitte um Hilfestellung in einer problematischen Situation wendet. Diese Beratung kann institutionalisiert sein oder nicht, die rater- teilende Person kann ein Experte / eine Expertin sein bzw. eine einschlägige Ausbildung haben oder auch nicht.“

Dass auch (informierte) Laien als Ratgeber fungieren, ist also nichts Neues (Antos 2001:21), und vor allem bei Hilfeforen von Selbsthilfegruppen kann es passieren, dass Nicht-Experten sich gegenseitig helfen, d.h. sich Ratschläge geben. Manchmal fehlt die Kontrolle durch Experten/innen überaus, die im IGAN-Forum dagegen garantiert ist. Fachleute sollen hier allerdings nicht lediglich den Informationsfluss überwachen, was noch den Textsortenkonventionen entspräche. Fast revolutionär klingt folgende Aussage von Guido Fluri: „Mit Tausenden von Betroffenen-Berichten nutzen auch immer mehr Mediziner die Hinweise der Betroffenen über das Forum." Mit anderen Worten profitieren selbst Ärzte vom Informationsaustausch, Experten und Laien lernen voneinander, weil sie über Kenntnisse verfügen, die sich wechselseitig ergänzen (sollen), um das Akustikusneurinom besser verstehen zu können, was das gemeinsame Ziel darstellt. Die Feststellung, dass die tradierten starren Rollen nunmehr aufgebrochen sind, wird durch einen Forumsbeitrag bestätigt, dessen Verfasser zugleich Arzt und Patient ist:

Selbst als Kollege habe ich so viele unterschiedliche Zahlen und Meinungen gehört. Im Forum haben viele Beiträge und Zahlen mein Verständnis erheblich gefördert. Ich hoffe, dass wir notgedrungen durch unsere Beschwerdebilder weiter dazu beitragen können. (wally, 06.05.2012) 
Interessant ist im letzten Satz das inklusive „wir“, das sich auf die Gemeinschaft der Patienten bezieht, vielleicht ein Zeichen dafür, dass sich wally hier mehr Patient als Arzt fühlt. Allerdings vereinigen sich in ihm das Fachwissen des Arztes und das Allgemeinwissen des Patienten, die normalerweise in verschiedenen Personen, also getrennt aufbewahrt sind. Der Informationsaustausch im Forum erweist sich auch als ein Versuch, diese Wissenskluft zu überbrücken, aber dazu ist eine erfolgreiche Kommunikation vonnöten.

\section{Forum als Informationsplattform}

Forumsnutzer müssen sich immer an Verhaltens- bzw. Kommunikationsregeln halten, die aber oft nicht so klar und deutlich zur Sprache kommen wie im IGAN-Forum, in dem insbesondere der Moderator ANFux eine Art Netiquette entwickelt, damit eine effektive Wissensvermittlung erfolgt. Seine Anweisungen sind vor allem in einem Beitrag vom 11.02.2012 enthalten, der mehrmals im Forum erscheint, in allen elf Rubriken ganz oben als erstes Thema, wie in der folgenden Abbildung zu sehen ist:
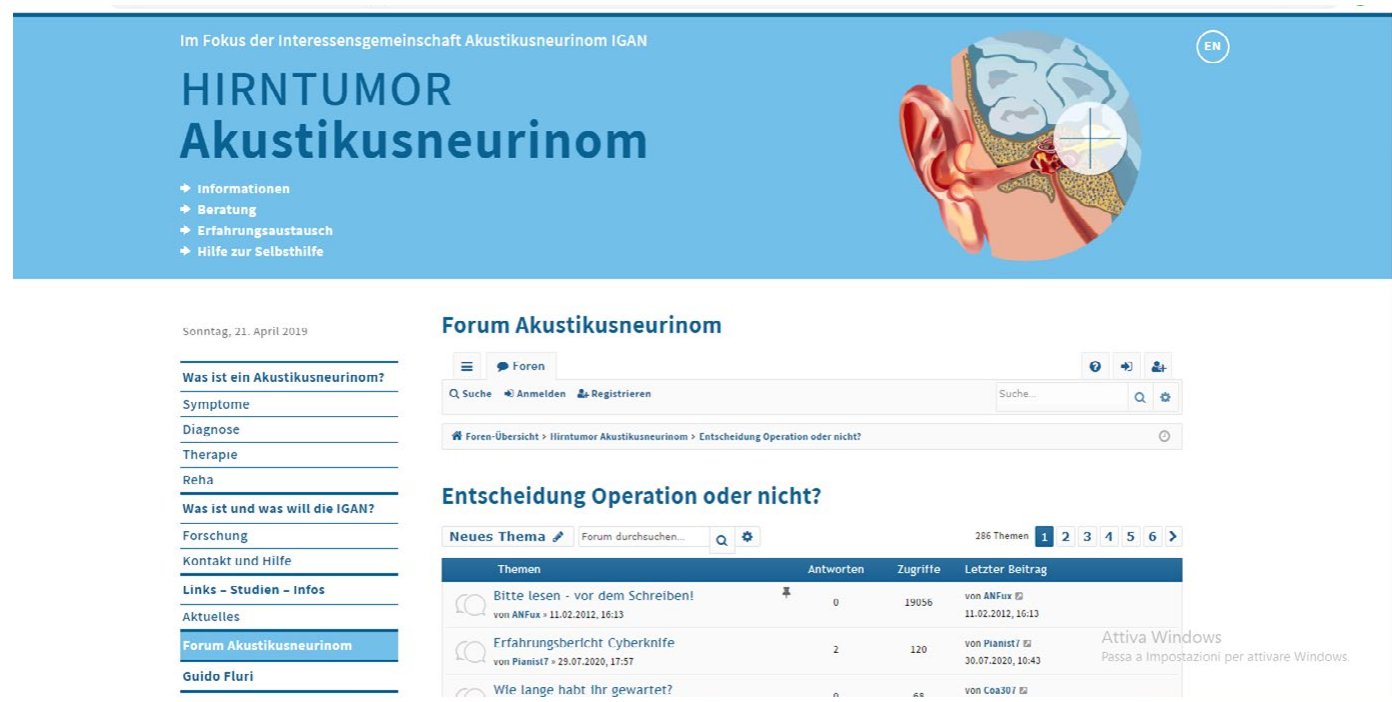

Abb. 1: Rubrik ,Entscheidung Operation oder nicht?‘ des IGAN-Forums (URL 9)

Das Thema heißt „Bitte lesen - vor dem Schreiben!“ und umfasst zwei Arten Empfehlungen an die Forumsmitglieder, die einerseits auf eine allgemeine Regel des gesunden Menschenverstandes (zuerst lesen, dann schreiben) hingewiesen, andererseits mit tiefgehenden Überlegungen zur Spezifik des Forums als Informationsplattform konfrontiert werden.

\subsection{Zuerst lesen, dann schreiben}

Nichts anderes als Commonsense sind die Aufforderungen von ANFux, zuerst die schon veröffentlichten Postings zu lesen und erst danach ein neues Thema zu eröffnen, falls die gesuchten Informationen nicht gefunden worden sind. Das Forum ist außerdem gut überschaubar, denn es ist mit einer Suchfunktion versehen und notfalls werden Beiträge neu zugeordnet bzw. an andere Stellen platziert, damit die Übersichtlichkeit erhalten bleibt.

Übrigens ist das Forum nicht die einzige Informationsquelle auf der IGAN-Plattform, ihm vorangestellt ist nämlich eine Reihe von sogenannten Themenseiten, die so systematisch wie laien- 
gerecht sehr ausführliche Auskünfte über das Akustikusneurinom erteilen. Mit den Themenseiten sollte man auf jeden Fall anfangen, um sich auf die Inhalte des Forums vorzubereiten.

\subsection{Hierarchie der Textfunktionen}

Wenn der Forumsnutzer nach gründlichem Lesen der vorliegenden Texte auch mal zum aktiven Beitragsschreiber werden will, empfiehlt ihm ANFux, die besondere Zielsetzung des Forums zu beachten, das er - getreu dem Betreiber - als „Informationsplattform“ konzipiert. Dieses Anliegen von ANFux kann mittels der Sprachfunktionen im Sinne von Jakobson (1960) erläutert werden, obwohl der Moderator als linguistischer Laie nie von „Textfunktionen“ spricht. Rein intuitiv erkennt er drei Funktionen im Forum, phatische, emotive und referentielle, wobei die letztere die allerwichtigste sei.

\subsubsection{Die phatische Funktion}

Die Pflege sozialer Kontakte erfolgt durch sprachliche Mittel wie Anrede, Grußformel und Unterschrift, die nach dem Moderator nie fehlen dürfen, ,wie es beim Chatten üblich sein kann“. Auf die phatische Funktion ist auch Small-Talk zurückzuführen, also Glückwünsche vor einer Operation, Gratulationen danach, Höflichkeiten im Allgemeinen, die den menschlichen Beziehungen guttun und ins Forum hineingehören, solange sie nicht zu viel Platz einnehmen bzw. von der informativen Hauptfunktion der Texte ablenken: „In einigen Situationen, wie bspw. dem Gratulieren nach einer Operation oder dem ,Alles Gute' am Vortag der OP kann man und sollte man auch mit ein paar Sätzen plaudern“, gibt ANFux zu, aber das Forum soll in erster Linie auf den Wissenstransfer ausgerichtet bleiben. Als Nebenfunktion, die nicht nur akzeptabel, sondern sogar erwünscht ist, gilt auch die emotive Funktion, unter der Bedingung, dass sie sich innerhalb bestimmter Grenzen hält.

\subsubsection{Die emotive Funktion}

„Angst“" ist eines der Wörter, die im Forum am meisten vorkommen, mehrmals wiederholt, gesteigert durch Adjektive und Adverbien sowie verstärkt durch Ausrufezeichen und Großschreibung wie im folgenden Beitrag, in dem die emotive Funktion brisant hervortritt:

Ich war total verwirrt, ich? ich bin doch erst 20 ? ich rauche nicht, trinke fast nie? ich mache regelmäßig Sport. DAS KANN NICHT SEIN? Am meisten beschäftigt mich die Angst gerade. Es macht mir wirklich höllische Angst. (Rayisa, 29.06.2015)

Als Mensch und Betroffener zeigt ANFux ein gewisses Verständnis für solche Angstausbrüche, denn er weiß wohl, was für Emotionen und Gefühle ein Akustikusneurinom mit sich bringt, trotzdem fühlt er sich als Moderator gezwungen, immer wieder auf das Informationsgebot zu bestehen. Er sieht nämlich eine exzessive Emotivität als Gefahr, die den Hauptzweck der Informationssuche zu beeinträchtigen droht.

\subsubsection{Die referentielle Funktion}

Wenn die Angst immer ein schlechter Ratgeber ist („Das ist nicht nur ein wohlfeiler Spruch“, elf, 12.05.2016), bedarf eine fachliche Beratung einer gewissen Vorbereitung, um zustande kommen zu können:

Wer geht zum Arzt, ohne sich bewusst gemacht zu haben, wie er dem Arzt sein Problem schildert? Wohl kaum jemand. Wie bereitet man sich darauf vor? Am besten mit schriftlichen Notizen. Was sollte man notieren? Alles Abnormale, das erstmalige Spüren, die weitere Entwicklung, Zeit und Stärke von Symptomen, eben alles, was einen bewegt. Fragen, ganz ehrlich alle, die einen bedrängen, möglichst geordnet. Und nicht anders ist es, wenn man Rat in einem medizinischen Forum sucht!! (ANFux, 11.02.2012) 
Ein wissenschaftliches Denken auf empirischer Basis wird hier von Ratsuchenden verlangt, die in der Beschreibung ihrer Beschwerden um Sachlichkeit und Präzision bemüht sein sollen, damit ihnen mit nützlichen Informationen geholfen werden kann. Mit anderen Worten tragen zur Selbsthilfe des Mottos nur Postings bei, die ihrerseits schon einen hohen Grad an Informativität aufweisen und auf diese Weise dementsprechend gezielte Antworten ermöglichen.

Die Nutzer finden in den Themenseiten zuerst mal allgemeine Informationen und können dann, wenn nötig, durch die Interaktion im Forum auf sie zugeschnittenes Fachwissen erwerben. „Das passgenaue Zuschneiden von Botschaften auf die Besonderheiten und Bedürfnisse des Einzelnen“ nennen Rossmann/Stehr ,Tailoring“ (2010:353).

Wie gesagt legt ANFux sein besonderes Augenmerk auf die erste Stufe des Wissenstransfers, auf das Fragen, und verdeutlicht durch ein negatives Beispiel, das er extra zu diesem Zweck erfunden hat, die kommunikativen Defekte mancher Beiträge:

„Seit zwei Tagen weiß ich, dass ich einen Tumor habe. Ich bin völlig kopflos. Was kann ich jetzt tun?

Gibt es in der Nähe meines Wohnortes eine gute Klinik? “(ANFux, 11.02.2012)

Besonders auffällig sind hier die terminologische Ungenauigkeit und die Dominanz der emotiven statt der referentiellen Funktion. Das angegebene Beispiel ist mit einem Kommentar versehen, der die Folgen seiner kommunikativen Defizite hervorhebt:

Was passiert nach so einem Beitrag? Es beginnt ein langes Ping-Pong zwischen Forumsmitgliedern, die helfen wollen und dem Ratsuchenden, dem Stück für Stück die Informationen aus der Nase gezogen werden müssen, die man braucht, um ihm einen qualitativ hochwertigen Rat geben zu können.

(ANFux, 11.02.2012)

Die Kommunikationsstörung, die ANFux feststellt, besteht im Grunde genommen in einem Verstoß gegen die Textsortenkonventionen, die in ihm einigermaßen intuitiv vorhanden sind, obwohl er auch hier nie den textlinguistischen Terminus „Textsorte“ verwendet (vgl. Lux 1981:4 f.; Sandig 1987:120). Am Anfang des genannten Beitrags „Bitte lesen - vor dem Schreiben!“ steht folgende Anmerkung: „Das IGAN-Forum ist kein Chatroom für Plaudertaschen. Es ist eine Informationsplattform für wirklich Ratsuchende.“ Dadurch, dass „Chatroom“ und „Informationsplattform“ fett gedruckt sind, wird die implizite Gegenüberstellung der Textsorten ,Plauder-Chat" und ,Hilfeforum “ unterstrichen, die sowohl inhaltlich als auch stilistisch verschiedene Merkmale aufweisen.

Die terminologische Ungenauigkeit und das Vorherrschen der phatischen und der emotiven Funktion setzt ANFux in Verbindung mit Chats, während die referentielle Funktion und die entsprechende Ausdrucksweise typisch für das Forum sind. Mit „Plaudertaschen“ meint der Moderator Forumsmitglieder, die im Forum plaudern möchten, als wäre es ein Chat: Ihr misslungenes Kommunikationsverhalten veranlasst ihn dazu, an alle zu appellieren mit der Bitte um Einhaltung der Textsortenkonventionen, die auch Stilnormen mit einbeziehen: ,[...] appelliere ich an alle Nutzer des Forums, den Charakter des Forums als Informationsplattform zu einem ernsten Thema zu wahren, das auch in einem angemessenen Stil.“

Die appellative Textfunktion, die durch das performative Verb ,appellieren' signalisiert wird, bleibt ihm als Moderator vorbehalten, denn ansonsten beabsichtigen die Beiträge der anderen Verfasser keine besondere Wirkung jenseits des regen Informationsaustausches, der von vornherein zu den Zielsetzungen des Forums gehört. Das Antworten als erwartete Reaktion auf das vorherige Fragen ist für das Forum textkonstitutiv, ganz anders als die gelegentlichen Persuasionsversuche, die ab und zu im Forum auftauchen, die sehr appellierend klingen und eben deswegen von den Moderatoren getadelt werden, weil jeder am Ende für sich entscheiden soll.

\subsection{Laienhaftes Textsortenwissen}

In seinem Appell skizziert ANFux die Grundrisse einer textlinguistischen Unterscheidung, die weit über die Textfunktion hinausgeht; was er unter „einem angemessenen Stil“ versteht, kann 
nämlich aus dem Vergleich mit der Chat-Plauderei herausgearbeitet werden, die ANFux für die Chat-Kommunikation schlechthin hält. Die Realität sieht viel komplexer aus: „Chats sind Kommunikationsformen, die je nach Teilnehmergruppe, kommunikativer Zwecksetzung und Thema für unterschiedliche Funktionen genutzt werden können und auch sprachlich unterschiedlich geprägt sein können“ (Fandrych/Thurmair 2011:284), was ohnehin auch für Foren gilt, denn wie schon erwähnt sind nicht alle Foren Hilfeforen.

Die „vorwiegend phatisch-unterhaltenden Plauder-Chats“ (Fandrych/Thurmair 2011:284), die ANFux mit seinen Anmerkungen meint, sind nur eine markante Ausprägung des Chats, dessen prototypische Erscheinung (Spitzmüller 2009:79-80), die linguistische Laien intuitiv vor Augen haben, wenn sie an Chats denken. Diese, wenn auch naive, jedoch weitverbreitete Auffassung von Chats kann herangezogen werden, um im Gegensatz dazu bestimmte Eigenschaften der medizinischen Internetforen besser herauszuarbeiten.

Als Ausgangspunkt für den Vergleich kann der Begriff der konzeptionellen Mündlichkeit dienen. In Anlehnung an Koch/Oesterreicher (1986) äußert sich Ehrhardt folgendermaßen zu Foren: „Es handelt sich medial um geschriebene Kommunikation, konzeptionell lassen sich aber durchaus auch Merkmale von Mündlichkeit finden“ (Ehrhardt 2010:177). Das gilt umso mehr für Chats, die ein Plus an Mündlichkeit aufweisen, so dass sie als ,getippte Gespräche“ (Storrer 2001) definiert worden sind.

Gesprochensprachliche Merkmale wie Tilgungen, Klitisierungen, Assimilationen und Regionalismen sind typisch für Chats (Fandrych/Thurmair 2011:298), in Foren allerdings seltener, wie auch im IGAN-Forum festgestellt werden kann. Auch elliptische Sätze sind in Foren nicht so massiv vorhanden wie in Chats, was in den asynchronen Rezeptions- und Produktionsbedingungen begründet ist:

„Unmittelbare Antworten können vorkommen, sind aber nicht die Regel und werden von Nutzern auch nicht im gleichen Ausmaß wie im Falle von Chats erwartet. Die Teilnehmer von Foren können sich also vergleichsweise viel Zeit für die Planung und Formulierung ihrer Beiträge nehmen“ (Ehrhardt 2009:138).

Eben aus diesem Grund fällt im IGAN-Forum die nähesprachliche Stilprägung der Spontaneität weg, die Beiträge sind in den meisten Fällen wohl überlegt und durchschnittlich länger oder sogar viel länger als in Chats, orthographische Normen werden im Grunde genommen beibehalten und auch sonstige Kennzeichen der Schriftsprache sind anzutreffen, unter anderem eine geordnete Argumentationsfolge, die bei einigen Beitragenden besonders markant ist. Im Kontinuum zwischen Mündlichkeit und Schriftlichkeit stehen Foren dem Schriftlichkeitspol sowieso etwas näher als Chats, und noch näher dann, wenn wir medizinische Hilfeforen und Plauder-Chats als respektive Vertreter betrachten.

Exemplifiziert wird der angemessene Stil, für den ANFux plädiert, gerade durch seine Postings, in denen er eine durchaus korrekte, aber noch gemeinsprachliche Syntax mit einem teilweise sehr fachlich ausgeprägten Wortschatz verbindet. Dass diese Postings einen vorbildlichen Charakter haben, erweist sich aus der relativ hohen Einheitlichkeit im sprachlichen Ausdruck, die im Forum tatsächlich zu bemerken ist: Die Laien haben die Fachlexik von den Ärzten übernommen, die ihrerseits - bewusst oder unbewusst - auf die Verdichtung der hochspezialisierten Fachtexte verzichten (auch in ihren Beiträgen fehlen weitgehend typische Formen des Nominalstils wie erweiterte Partizipialattribute).

Im IGAN-Forum ist eine Online-Community tätig, die sich auch stilistisch eine eigene Identität erschaffen hat. Aus den programmatischen Richtlinien von ANFux lässt sich folgender Schluss ziehen: Da die Wissensvermittlung über die Sprache erfolgt, sind auch für einen Laien linguistische Betrachtungen zu einer effektiven Wissensvermittlung unentbehrlich. Deswegen geht das Bemühen um sprachliche Sorgfalt Hand in Hand mit dem Aufbau des Forums als Wissensplattform. 


\section{Fazit und Ausblick}

Das Internetforum ist nicht per se ein geeigneter Ort für den Wissenstransfer. Es kann unter bestimmten Umständen ein solcher werden, sofern sich die Nutzer an die kommunikativen Regeln halten, die ANFux in seinem griffigen Beitrag so laienhaft wie klar darstellt. Das neue Medium bietet bloß neue Möglichkeiten, die die Gemeinschaft der Forumsteilnehmer zu ihren Zwecken nutzt.

Die Untersuchung des IGAN-Forums hat bei aller Beschränktheit des verwendeten Korpus den Weg zur Herausbildung einer neuen Textsorte gezeigt, die medizinisches Hilfeforum genannt werden kann und jünger ist als andere Erscheinungen derselben Kommunikationsform, wie z. B. das Diskussionsforum, das schon öfter Forschungsgegenstand von textlinguistischen Analysen war. Während das Internetforum ,allein durch situative bzw. mediale Merkmale definiert“ (Brinker 2001:139), also eine Kommunikationsform ist, ist die auf Wissensvermittlung abzielende Textsorte der medizinischen Hilfeforen auch kommunikativ-funktional und sprachthematisch festgelegt. So weit textklassifikatorisch, wobei nur noch zu bemerken bleibt, dass die hier umrissenen Beobachtungen anhand von vielen weiteren Textexemplaren überprüft werden sollten, zudem Online-Textsorten stets im raschen Wandel begriffen sind. Erst weitere Untersuchungen können den vorgeschlagenen Klassifikationsversuch bestätigen oder relativieren.

Die Relevanz der Wissensvermittlung im IGAN-Forum eröffnet auch aus soziologischer Sicht neue Perspektiven. Kein Wunder, denn im raschen Wandel ist nicht zuletzt die Gesellschaft, die online kommuniziert. Textsorten sind soziale Phänomene, die mit der Entwicklung der Gesellschaft interagieren und diese Entwicklung sogar vorantreiben können. Das Forum zeugt von einem neuen Verhältnis zwischen Ärzten und Patienten, das nicht mehr hierarchisch konzipiert wird. „Diese Veränderung im Arzt-Patienten-Verhältnis wird als Wandel von der patriarchalischen Medizin zur partnerschaftlichen Medizin bezeichnet“ (Wiese 2001:227). Eckkrammer (2001:400) spricht in diesem Sinne von einer „Demokratisierung des medizinischen Wissens“, das notwendigerweise mit seiner Popularisierung einhergeht. Das IGAN-Forum beweist diesen Trend, aber manchmal reicht es weit darüber hinaus und schlägt epistemologisch neue Bahnen.

ANFux schreibt: „Wenn wir als Selbsthilfevereinigung ein respektierter Partner der Ärzte sein wollen, und für die AN-Ratsuchende eine echte Hilfe, dann müssen wir uns weiterbilden“ (14.10.2010). Mit anderen Worten sollen sich betroffene Laien um Wissenserwerb bemühen, wenn sie die eigene Krankheit in den Griff bekommen wollen. Ihre Annäherung an das Fach erfordert allerdings von der Arztseite die Verständlichmachung der fachlichen Inhalte durch populärwissenschaftliche Strategien wie die Reduktion der Informationsfülle, die im Forum wie oben angedeutet durch einen niedrigen Verdichtungsgrad der Informationen auf syntaktischer Ebene erreicht wird.

Obwohl die Beziehung zwischen den Interaktanten als partnerschaftlich beschrieben wird, unterstreichen diese Worte von ANFux de facto die wissensbasierte Überlegenheit der Fachleute, die den Laien sozusagen eine helfende Hand reichen, damit sie ein höheres Wissensniveau erlangen und dann als informierte Laien auch ihren Leidensgenossen helfen können. Bisher haben alle Untersuchungen zur Fachkommunikation zwischen Experten und Laien diese Vertikalität des Fachwissens als selbstverständlich vorausgesetzt (vgl. beispielsweise Busch 1994 und Wichter 2009).

Im IGAN-Forum kommt überdies eine andere Vorstellung ansatzweise zum Vorschein, die das Fachwissen der Experten und das Alltagswissen der Laien (vgl. Palm 2001:349) gegenüberstellt, ohne jedoch das letztere als minderwertig zu betrachten, ganz im Gegenteil: Als zwei unterschiedliche Wissenssysteme sollen sie im Forum zusammentreffen. Die Wissenskluft wird nicht negiert, aber anders, sozusagen horizontal, gedeutet, indem das Potential des Laienwissens betont wird. An folgender Stelle statuiert ANFux selbst die Wissenschaftlichkeit des Alltagswissens, wenn solches Alltagswissen von Betroffenen „nüchtern, analytisch und konstruktiv“, also emotionslos dargelegt wird:

Das Notieren aller Auffälligkeiten, die später als Symptome bezeichnet werden, den Zeitpunkt des ersten und ihres wiederholten Auftretens, die Begleitumstände, die Beeinflussbarkeit, die Heftigkeit und Dauer - kurz, alles, was zur Beschreibung und Charakterisierung beitragen kann, ist es wert, 
notiert zu werden. Warum? Es hilft dem Arzt, die Krankheit, d.h. ihr Stadium, ihre Schwere, einzuschätzen, einzustufen. Und das hilft auch dem Patienten. Ich sage immer salopp, rd. 50\% des Wissens erlangt der Arzt vom Patienten. Je mehr der Patient sich notiert, um so größer ist absolut dieser Anteil. (ANFux, 24.08.2009)

ANFux und wally, beide zugleich Ärzte und Betroffene, halten das Allgemeinwissen der Patienten für so beachtenswert, dass es ihrer Ansicht nach die Schwerpunkte der medizinischen Forschung und Praxis mitbestimmen sollte:

Ich bin überzeugt, dass die Zeit reif ist, Schwindel zu einer ernst zu nehmenden Beeinträchtigung der Lebensqualität zu erheben. Das ist unsere Forderung, die der Patienten. Das anzuerkennen, ist Sache der Ärzte und Therapeuten. Und Lösungen suchen, Schwindel zu beseitigen und in manchen Situationen gar nicht erst entstehen zu lassen - das ist unser aller Anliegen. Wobei wir beobachten, mitdenken und mitmachen müssen, die entscheidenden Schritte sind jedoch von den Ärzten zu erhoffen. (ANFux, 06.12.2010)

Ich bin nach vielen Gesprächen mit Neurologen und Neurochirurgen der Meinung, dass so ,subjektive' Beschwerden wie Kopfschmerz, Schwindel, Tinnitus und Hyperakusis dringend in den Fokus der postoperativen Verlaufsbeobachtung gestellt werden muss [sic!]. [Unterstreichungen im Original] (wally, 27.10.2012)

Seitdem wally an dieser seltenen Krankheit leidet, kann er sich in seine Patienten einfühlen, was früher überhaupt nicht der Fall war: Kurz zu mir: ich bin selber Allgemeinarzt in eigener Praxis und erlebe seit vielen Jahren, komische Menschen und Geschichten'. Nun fühle ich mich selber komisch und erkenne die Schwierigkeit dies zu benennen [...] (03.10.2010). Seine Einstellung hat sich so grundlegend verändert, dass er jetzt die Erfahrung der Betroffenen, d.h. ihre Wahrnehmung der Beschwerden, eher als einen Wissensschatz als eine Hürde ansieht.

Es gilt also, um die Krankheit besser zu verstehen, diesen Wissensschatz nicht unberücksichtigt zu lassen, und vor wichtigen Entscheidungen mal auch auf das „Bauchgefühl“ zu hören, das wally als ,verlässlich[es]“ qualifiziert. Die Anerkennung des Bauchgefühls als Wissensgrundlage ist ohne Zweifel der bedeutendste Ausdruck des Patienten-Empowerments, das zuerst im Online-Forum nicht in der reellen Welt - stattfindet und sich von da aus auch in der reellen Welt immer mehr ausbreiten wird.

\section{Literaturverzeichnis}

\section{Primärliteratur:}

Internetportal und Forum zum Hirntumor Akustikusneurinom. Im Fokus der Interessengemeinschaft Akustikusneurinom IGAN. https://www.akustikusneurinom.info [09.01.2021].

\section{Sekundärliteratur:}

AnTos, Gerd (2001): Transferwissenschaft. Chancen und Barrieren des Zugangs zu Wissen in Zeiten der Informationsflut und der Wissensexplosion. In: Wichter, Sigurd / ANTOS, Gerd (Hrsg.): Wissenstransfer zwischen Experten und Laien. Umriss einer Transferwissenschaft. Bern, S. 3-33.

Berg, Volker (2010): Medizinische Internetforen: Ärzte als kompetente Teilnehmer. In Deutsches Ärzteblatt, Jg. 107, Heft 45. Zugänglich unter: https://www.aerzteblatt.de/archiv/79214/Medizinische-Internetforen-Aerzte-als-kompetente-Teilnehmer [09.01.2021].

Born, Günter (2010): Nett, Sie online zu treffen. München.

BrinKER, Klaus (2001): Linguistische Textanalyse. Eine Einführung in Grundbegriffe und Methoden, 5., durchgesehene und ergänzte Auflage. Berlin.

Busch, Albert (1994): Laienkommunikation: Vertikalitätsuntersuchungen zu medizinischen Experten-Laien-Kommunikation. Frankfurt am Main. 
CZAuderna, André (2014): Lernen als soziale Praxis im Internet: Objektiv hermeneutische Rekonstruktionen aus einem Forum zum Videospiel Pokémon. Wiesbaden.

EBNER, Michael (2008): Internetforen: verwenden - einrichten - betreiben. Norderstedt.

EcKKrammer, Eva Martha (2016): Medizin für den Laien: Vom Pesttraktat zum digitalen Ratgebertext. Berlin.

EHRHARDT, Claus (2009): „Internetforen: Kommunikation und Diskussionskultur oder „Forenbeiträge schreiben ist quasi das fast-Food der Schreiberei“. In: Moraldo, Sandro (Hrsg.): Internet. kom. Neue Sprach- und Kommunikationsformen im WorldWide Web. Roma, S. 109-155.

EHRHARDT, Claus (2010): „Internet-Diskussionsforen: Eine Kommunikationsform im deutsch-italienischen Vergleich“. In: Foschi Albert, Marina / Hepp, Marianne/ Neuland, Eva / Dalmas, Martine (Hrsg.): Text und Stil im Kulturvergleich. Pisaner Fachtagung zu interkulturellen Wegen Germanistischer Kooperation. München, S. 170-191.

FANDRYCH, Christian / THURMAIR, Maria (2011): Textsorten im deutschen. Linguistische Analysen aus sprachdidaktischer Sicht. Tübingen.

Hass, Jan (2014): Social Media - Die Macht der Internetforen in der Caravanbranche. Eine Analyse der Relevanz und des Nutzungsverhaltens von Endkunden. Hamburg.

JAKobson, Roman (1960): Closing Statements: Linguistics and Poetics, in Style in Language, edited by Thomas A. Sebeok, Cambridge, Massachusetts, S. 350-377.

KLEINKE, Sonja (2015): „Internetforen: Laiendiskurs Gesundheit“. In: Busch, Albert / Spranz-FoGASY, Thomas (Hrsg.): Handbuch Sprache in der Medizin. Berlin; Boston, S. 405-422.

Koch, Peter / Oesterreicher, Wulf (1986): Sprache der Nähe - Sprache der Distanz. Mündlichkeit und Schriftlichkeit im Spannungsfeld von Sprachtheorie und Sprachgeschichte. In: Romanistisches Jahrbuch, Jg. 36, S. 15-43.

KRÜGER-BRAND, Heike E. (2010): Gesundheitskommunikation: Internet wichtigster Infokanal. In: Deutsches Ärzteblatt, Jg. 107, Heft 30. Zugänglich unter: https://www.aerzteblatt.de/ archiv/77740\#lit [09.01.2021].

KRÜGER-Brand, Heike E. (2012): Online-Arztpraxis: Virtuelle Sprechstunde per Video-Chat. In: Deutsches Ärzteblatt, Jg. 109, Heft 47. Zugänglich unter: https://www.aerzteblatt.de/ archiv/132853/Online-Arztpraxis-Virtuelle-Sprechstunde-per-Video-Chat [09.01.2021].

Lux, Friedemann (1981): Text, Situation, Textsorte. Probleme der Textsortenanalyse, dargestellt am Beispiel der britischen Registerlinguistik. Mit einem Ausblick auf eine adäquate Textsortentheorie. Tübingen.

MAGRIS, Marella (2009): Medizinische Fachbegriffe in der populärwissenschaftlichen Vermittlung: Ein Vergleich zwischen Deutsch und Italienisch. In: TaIno, Piergiulio / Brambilla, Marina/ BRIEST, Tobias (Hrsg.), Eindeutig uneindeutig. Fachsprachen - ihre Übersetzung, ihre Didaktik. Frankfurt am Main, S. 89-103.

PALM, Jörg (2001): Wissenstransfer zwischen Experten und Laien in Beratungsgesprächen. In: Wichter, Sigurd / Antos, Gerd (Hrsg.): Wissenstransfer zwischen Experten und Laien. Umriss einer Transferwissenschaft. Bern, S. 347-364.

ReInBold, Fabian (2008): Internetforen: Der Aufstand der Web-Patienten. In Die Zeit, Nr. 14. Zugänglich unter: https://www.zeit.de/2008/14/T-Dienstleistungen [12.11.2029]

Rossmann, Constanze / Stehr, Paula (2010): Gesundheitskommunikation im Internet. Erscheinungsformen, Potenziale, Grenzen. In: SchweIGer, Wolfgang / Beck, Klaus (Hrsg.), Handbuch Online-Kommunikation. Wiesbaden, S. 338-363.

SANDig, Barbara (1987): Textwissen. Beschreibungsmöglichkeiten und Realisierungen von textmustern am Beispiel der Richtigstellung. In: EngelKamp, Johannes / LoREnz, Kuno / SANDig, Barbara (Hrsg.), Wissenspräsentation und Wissensaustausch. Interdisziplinäres Kolloquium der Niederländischen Tage in Saarbrücken April 1986. St. Ingbert, S. 115-155.

Shaffu, Marc, Die Rolle von Patienteninformationssystemen in der Arzt-Patient-Beziehung: eine Fallstudie auf Basis digitaler Medien in der Dermatologie, epubli. 
SPITZMÜLLER, Jürgen (2009): „Chat-Kommunikation: Interaktion im virtuellen Raum als multidisziplinäres Forschungsfeld“. In: Moraldo, Sandro (Hrsg.), Internet.kom. Neue Sprach- und Kommunikationsformen im WorldWide Web. Roma, S. 71-107.

StORRer, Angelika (2001): Getippte Gespräche oder dialogische Texte? Zur kommunikationstheoretischen Einordnung der Chat-Kommunikation. In: LeHr, Andrea / Kammerer, Matthias / Konerding, Klaus-Peter / Storrer, Angelika / Thimm, Caja / Wolski, Werner (Hrsg.): Sprache im Alltag. Beiträge zu neuen Perspektiven in der Linguistik. Herbert Ernst Wiegand zum 65. Geburtstag gewidmet. Berlin, S. 439-465.

URBANEK, Margarethe (2020): E-Rezept: Online-Arztpraxis wird an Apotheken angebunden. In: ÄrzteZeitung, 11.02.2020. Zugänglich unter: https://www.aerztezeitung.de/Wirtschaft/OnlineArztpraxis-wird-an-Apotheken-angebunden-406616.html [09.01.2021].

WIESE, Ingrid (2001): Aspekte des Wissenstransfers im Bereich der Institution Gesundheitswesen. In: Wichter, Sigurd / Antos, Gerd (Hrsg.): Wissenstransfer zwischen Experten und Laien. Umriss einer Transferwissenschaft. Bern, S. 3-33.

Wichter, Sigurd (1995): Vertikalität von Wissen. Zur vergleichenden Untersuchung von Wissensund insbesondere Wortschatzstrukturen bei Experten und Laien. In: Zeitschrift für germanistische Linguistik. Deutsche Sprache in Gegenwart und Geschichte, Jg. 23, Heft 3. Berlin, S. 284-313.

\section{Internetquellen:}

URL 1: https://www.medizin-forum.de [09.01.2021].

URL 2: www.med1.de [09.01.2021].

URL 3: www.docInsider.de [09.01.2021].

URL 4: www.onmeda.de [09.01.2021].

URL 5: www.wer-weiss-was.de [09.01.2021].

URL 6: https://www.akustikusneurinom.info/forum3/ [09.01.2021].

URL 7: https://www.akustikusneurinom.info/cms/Was-ist-und-was-will-die-IGAN/ [09.01.2021].

URL 8: https://www.akustikusneurinom.info/cms/Guido-Fluri/ [09.01.2021].

URL 9: https://www.akustikusneurinom.info/forum3/viewforum.php?f=1 [09.01.2021]. 